\title{
Real-Time Monitoring System and Advanced Characterization Technique for Civil Infrastructure Health Monitoring
}

\author{
V. Bennett, ${ }^{1}$ T. Abdoun, ${ }^{1}$ M. Zeghal, ${ }^{1}$ A. Koelewijn, ${ }^{2}$ M. Barendse, ${ }^{3}$ and R. Dobry ${ }^{1}$ \\ ${ }^{1}$ Department of Civil and Environmental Engineering, Rensselaer Polytechnic Institute, 110 8th Street, JEC 4049, Troy, \\ NY 12180, USA \\ ${ }^{2}$ Deltares, Stieltjesweg 2, P.O. Box 177, 2600 MH Delft, The Netherlands \\ ${ }^{3}$ NYSDOT Geotechnical Engineering Bureau, 50 Wolf Road, Albany, NY 12232, USA
}

Correspondence should be addressed to V. Bennett, bennev@rpi.edu

Received 2 December 2010; Accepted 18 March 2011

Academic Editor: Devendra Narain Singh

Copyright () 2011 V. Bennett et al. This is an open access article distributed under the Creative Commons Attribution License, which permits unrestricted use, distribution, and reproduction in any medium, provided the original work is properly cited.

Real-time monitoring of civil infrastructure provides valuable information to assess the health and condition of the associated systems. This paper presents the recently developed shape acceleration array (SAA) and local system identification (SI) technique, which constitute a major step toward long-term effective health monitoring and analysis of soil and soil-structure systems. The SAA is based on triaxial micro-electro-mechanical system (MEMS) sensors to measure in situ deformation (angles relative to gravity) and dynamic accelerations up to a depth of one hundred meters. This paper provides an assessment of this array's performance for geotechnical instrumentation applications by reviewing the recorded field data from a bridge replacement site and a full-scale levee test facility. The SI technique capitalizes on the abundance of static and dynamic measurements from the SAA. The geotechnical properties and constitutive response of soil contained within a locally instrumented zone are analyzed and identified independently of adjacent soil strata.

\section{Introduction}

The health and state of the aging and overburdened civil infrastructure in the United States has been subjected to renewed scrutiny over the last few years. The American Society of Civil Engineers reports that this state threatens the economy and quality of life in every state, city and town in the nation. As one example, the United States Army Corps of Engineers noted in early 2007 that nearly 150 United States levees pose an unacceptable risk of failing during a major flood [1].

Additionally, losses associated with failures of soil systems continue to grow in the United States and elsewhere in view of increased development in hazard-prone areas. The control and mitigation of the effects of these failures requires a better understanding of the field response of soil systems. In order to overcome these problems, the performance of these systems needs to be reliably predicted, and such predictions can be used to improve design and develop efficient remediation measures. The use of advanced in situ monitoring devices of soil systems, such as the shape acceleration array (SAA) system described in this paper, and the development of effective system identification and model calibration is essential to achieve these goals.

Soil and soil-structure systems are massive semi-infinite systems that have spatially varying parameters and state variables. These systems exhibit a broad range of complex response patterns when subjected to extreme loading conditions [2-4]. Accurate prediction of site response is essential in hazard analyses, health monitoring, or design of civil infrastructure systems. These predictions require the availability of calibrated and validated computational models [5]. Soil sample experiments (e.g., triaxial tests) have been widely used to evaluate the mechanical properties and calibrate constitutive relations of geotechnical systems. Nevertheless, because of limitations in reproducing in situ stress and porefluid conditions, the consensus is that these experiments may not fully reflect reality. Thus, fundamental differences still separate geotechnical engineering science and practice [6]. Peck [7] states that these differences stem from the fact that 
science relies on laboratory soil sample tests, while practice is rooted in field performance data and associated empirical studies. Consequently, some practitioners remain skeptical about models developed by geotechnical engineering scientists, for the obvious reason that very few models have been properly calibrated with field performance.

The answer to this challenge partly resides in the development of tools for short- and long-term health monitoring of existing civil infrastructure along with data reduction tools of systems identification and inverse problems. The knowledge gained from this monitoring and analysis would aid in planning for maintenance and rehabilitation of these infrastructure systems and will improve the design, construction, operation, and longevity. Critical soil-structure elements of the civil infrastructure which are important to monitor include bridge foundations, abutments, and support systems, retained, reinforced, or stabilized rock and earthen embankments and levees, slopes and mechanically stabilized earth (MSE) walls, and tunnels and tunnel linings. This paper presents a newly developed sensor array and local system identification technique. The array is capable of measuring in situ deformations and accelerations up to a depth of one hundred meters and is essentially an in-place inclinometer coupled with accelerometers. The frequency and spatial abundance of data made available by this new sensor array enables tools for the continuous health monitoring effort of critical infrastructure under a broad range of static and dynamic loading conditions.

The concept of the presented MEMS-based, in-place inclinometer-accelerometer instrumentation system is centered on measurements of angles relative to gravity, using triaxial MEMS (micro-electro-mechanical Systems) accelerometers, which are then used to evaluate inclinations (i.e., deformations). The same MEMS accelerometers also provide signals proportional to vibration during earthquakes or construction activities. Three accelerometers are contained in each $30 \mathrm{~cm}(1 \mathrm{ft})$ long rigid segment for measuring $x, y$, and $z$ components of tilt and vibration. The rigid segments are connected by composite joints that are designed to prevent torsion but allow flexibility in two degrees of freedom. These rigid segments and flexible joints are combined to form a sensor array. The system, called shape acceleration array (SAA), is capable of measuring three-dimensional (3D) ground deformations at $30 \mathrm{~cm}(1 \mathrm{ft})$ intervals and $3 \mathrm{D}$ acceleration at $2.4 \mathrm{~m}(8 \mathrm{ft})$ intervals to a depth of $100 \mathrm{~m}$ $(330 \mathrm{ft})$. The system accuracy of the SAA is $\pm 1.5 \mathrm{~mm}$ per $30 \mathrm{~m}$, an empirically derived specification from a large number of datasets. More detailed information on the design of the SAA is available in $[8,9]$.

The following sections present (1) a brief description of the SAA technology, (2) a case history of the application of the SAA system, both vertically and horizontally, at a bridge replacement site in New York, (3) a case history of the application of the SAA system at a full-scale levee testing facility in the Netherlands, and (4) a newly developed local system identification (SI) technique to analyze the response of active soil systems using the dense measurements provided by a network of SAAs. The developed SAA and local SI technique constitute a major step in the direction of

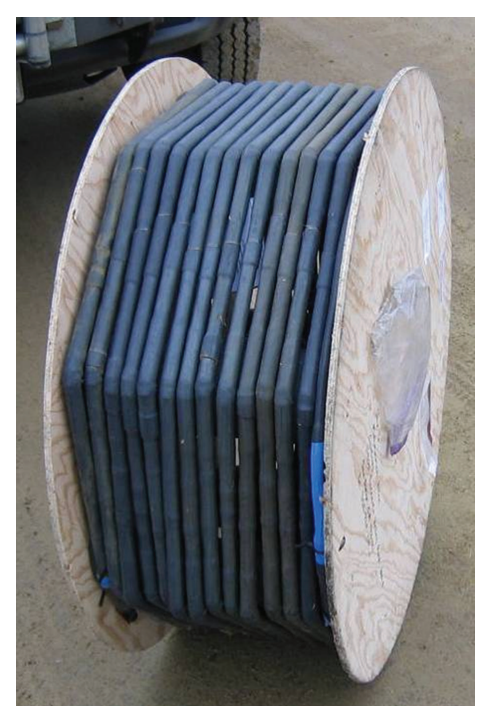

Figure 1: $32 \mathrm{~m}$ (104ft) SAA on shipping reel.

establishing long-term monitoring and analysis tools capable of providing a realistic picture of large deformation response and pending failure of soil and soil-structure systems.

\section{Sensor Description}

The SAA system uses temperature-calibrated MEMS accelerometers within $30 \mathrm{~cm}(1 \mathrm{ft})$ long rigid segments connected by composite joints that prevent torsion but allow flexibility in two degrees of freedom. The SAAs are factory-calibrated and completely sealed, requiring no field assembly or calibration. Because each segment of the SAA contains three orthogonal sensors, arrays can be installed vertically or horizontally as shown below in the New York State Department of Transportation (NYSDOT) bridge replacement case history. The intended array orientation does not need to be specified prior to installation. Orientation can be selected in the software. Each sensor has an output that is the sine of the angle of tilt over a range of 360 degrees. The sensor arrays are transported to the jobsite on an $86 \mathrm{~cm}$ (34 in) diameter reel, see Figure 1, and can be lowered into vertical, or pushed into horizontal, $25 \mathrm{~mm}$ ( $1 \mathrm{in}$ ) casing. The initial shape of the installation, or the absolute deviation of the installation from a virtual vertical or horizontal line, can be immediately viewed on a computer. An SAA is modeled as a virtual multisegment line in the software, with $x, y$, and $z$ data representing the vertices of this polyline. In the case of nearvertical installations, the vertices correspond to the joint centers of the array in 3D. For near-horizontal installations, the vertices show vertical deformation only versus horizontal position $[8,9]$.

Wireless SAA data transmission is made possible by the use of an on-site data acquisition system, called a wireless earth station. Similar to traditional probe and in-place inclinometers, data from the SAA represents deviations from a starting condition or initial reading. These data are sent wirelessly, over a cellular telephone network, to an automated 


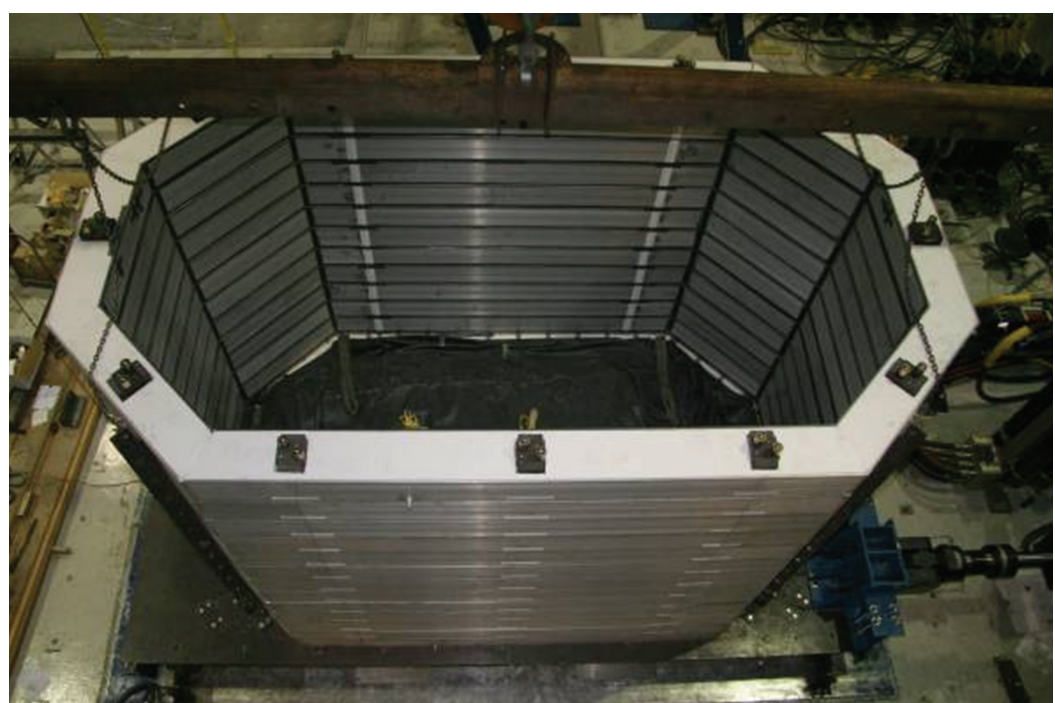

(a)

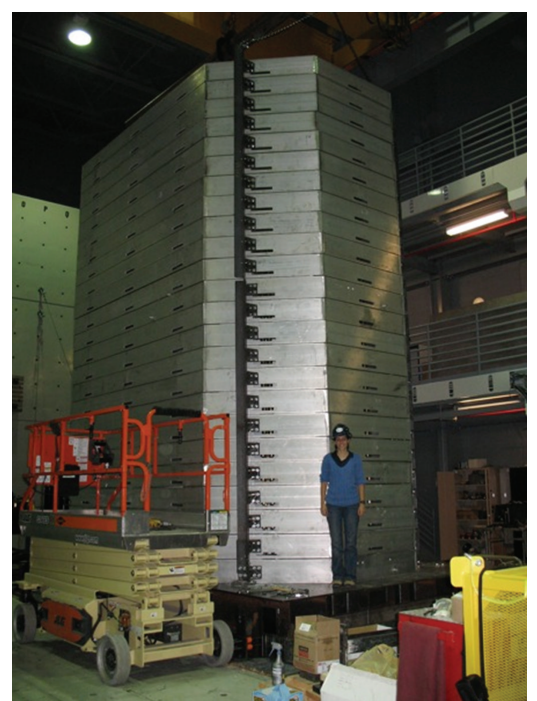

(b)

Figure 2: Assembly of laminar container at the University at Buffalo.

server, where data are made available to users through proprietary viewing software and an internet connection. Longterm system automated monitoring using SAAs typically collects data once or a few times a day, but this collection frequency can be respecified remotely by the user and changed at any time, through the same wireless interface used to receive the data. The SAA system is capable of collecting data at a sampling frequency rate of up to $128 \mathrm{~Hz}$, which makes it suitable for dynamic and seismic measurement. Each array is equipped with a trigger sensor that would automatically switch the SAA from slow to fast sampling rate in the case of a seismic event. Limiting the use of fast sampling rates to specific dynamic events significantly reduces the power consumption as well as data storage and transmission requirements.

The following section presents data that was collected during a full-scale lateral spreading experiment conducted in a laminar container at the University of Buffalo. The laminar container is $5 \mathrm{~m}(16.4 \mathrm{ft})$ long, $2.75 \mathrm{~m}(9.0 \mathrm{ft})$ wide, and $6 \mathrm{~m}$ $(19.7 \mathrm{ft})$ high and is capable of holding 150 tons of sand; see Figure 2 [10]. The results from two SAAs installed in this experiment provide an example of the range and type of data that can be collected by this system.

After this laminar container was instrumented and filled with loose sand and water, two 100-ton hydraulic actuators were used to induce predetermined motion with a $2 \mathrm{~Hz}$ frequency to the base of the box. The resultant soil liquefaction and lateral spreading was monitored using accelerometers within the soil deposit and on the ring laminates, potentiometers (displacement transducers) on the laminates, pore pressure transducers and two SAAs within the soil deposit. Each of the SAAs was $7 \mathrm{~m}$ (23.0 ft) long and contained 24 3D sensing elements. The acceleration and lateral displacement data from the SAA compared to the ring accelerometer and potentiometer data, respectively, are presented in Figure 3.
This data was collected during a sloping ground test, where the base of the box was inclined $2^{\circ}$.

At the end of the input shaking event, nearly the whole soil deposit was liquefied, and the ground surface displacement at the top of the laminar container had reached $32 \mathrm{~cm}$, as seen in Figure 3. Some discrepancies are observed between the SAA data and the ring accelerometer data after $6 \mathrm{~s}$, which is when the soil deposit began to liquefy. As the soil liquefied, the upper part of the SAA moved downslope with respect to the bottom of the array, thus the accelerometers were tilted with respect to their initial condition. This resulted in a slight DC component bias in the SAA acceleration readings. By filtering this low-frequency component, the acceleration readings from both types of instrumentation would match even more closely. Since this was a dynamic test, the dynamic component of the displacement was removed by filtering to obtain the results presented in Figure 3. This full-scale lateral spreading experiment provides a unique example of the simultaneous acceleration and permanent lateral displacement data captured by the SAA system. For more information on this full-scale experiment, see [11].

\section{SAA Field Installation at NYSDOT Bridge Replacement Site}

The SAA system was installed at a NYSDOT bridge replacement site over the Champlain Canal in upstate New York; see Figure 4. A brief site history and description of the installation process of the NYSDOT site is provided below along with a comparison between the vertical and horizontal SAA systems and traditional instrumentation, including a slope inclinometer and settlement plates. As shown in Figure 4, SP is settlement plates, SAAH is the horizontal SAA, SAAV is the vertical SAA, and PVDs are prefabricated vertical drains. 

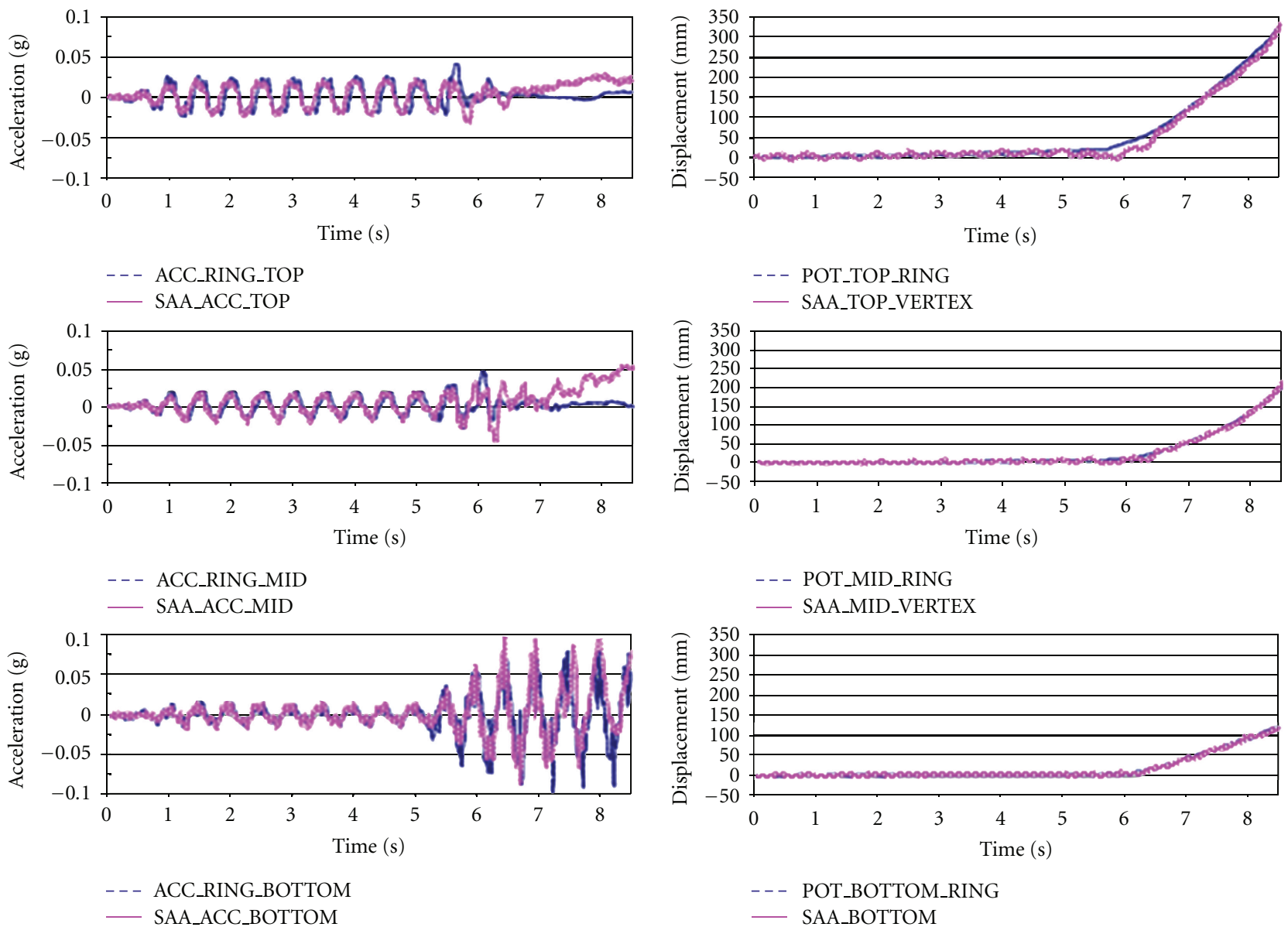

Figure 3: Acceleration ( $\mathrm{g}$ ) and lateral displacement comparison ( $\mathrm{mm}$ ) between SAA and laminar ring accelerometers and potentiometers, respectively, at the soil surface ( $0 \mathrm{~m}$ depth), mid-depth ( $2.8 \mathrm{~m}$ depth), and bottom of soil deposit (5.6 $\mathrm{m}$ depth); ACC_RING = Accelerometer on Laminar Ring, POT = potentiometer on laminar ring, SAA = shape acceleration array.

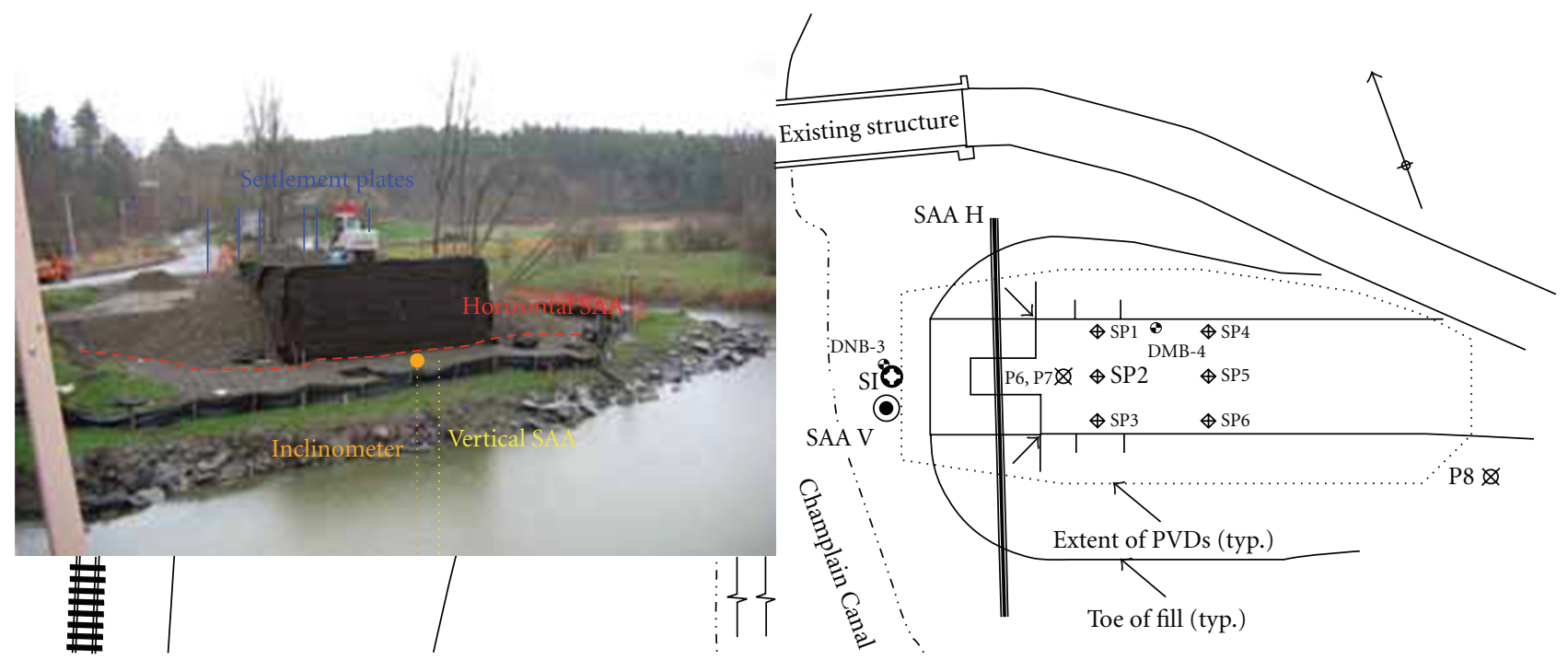

Figure 4: Bridge replacement and realignment over the Champlain Canal, NY. 


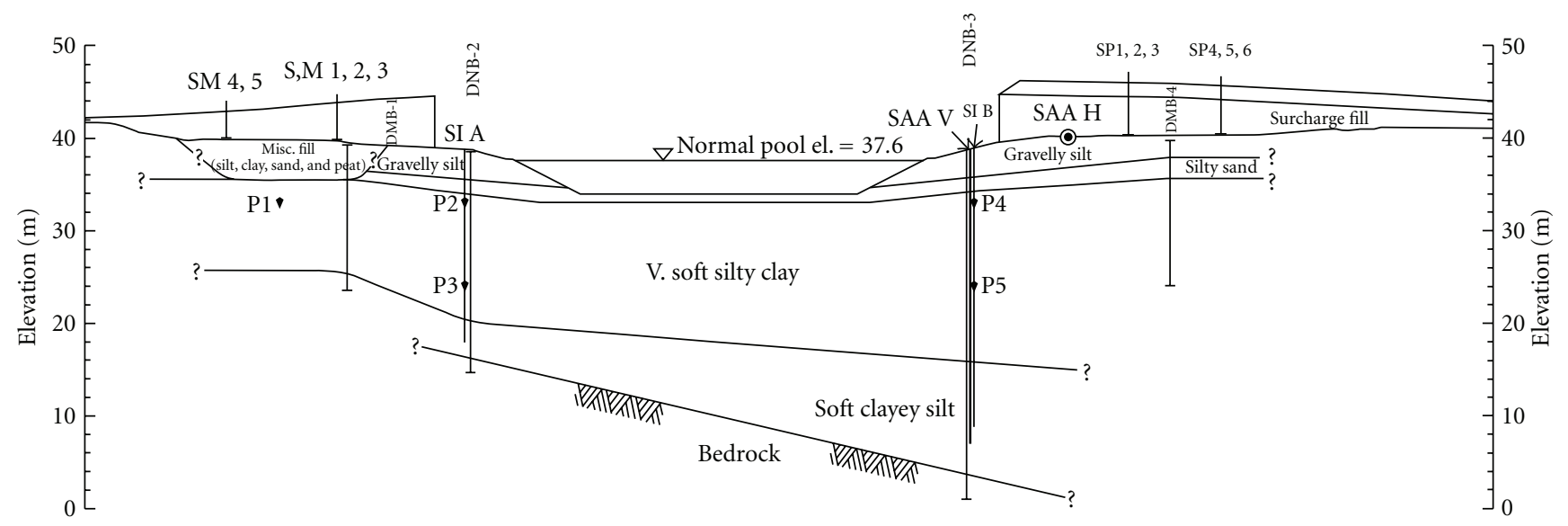

Figure 5: Soil profile and location of vertical SAA at Champlain Canal site.

The instrumentation plan for this site included the use of two $32 \mathrm{~m}$ (104 ft) long SAAs. One SAA was oriented horizontally and the other vertically to monitor the settlement and the lateral displacement, respectively, of a thirty-six meter deep soft clay deposit. Based on soil strength and consolidation testing performed on undisturbed boring samples, it was decided to employ prefabricated vertical drains (PVDs) and surcharge fills to accelerate the consolidation and strength gain of the clay layer prior to driving piles for the bridge.

The vertical SAA installed at this site was $32 \mathrm{~m}(104 \mathrm{ft})$ long, in order to extend below the very soft silty clay layer. The SAA was installed in a vertical borehole located approximately $3 \mathrm{~m}(9.8 \mathrm{ft})$ from the edge of the Champlain Canal and approximately $2.5 \mathrm{~m}(8.2 \mathrm{ft})$ from a traditional inclinometer casing, in the area between the surcharge fill and the canal; see Figure 5. A $50 \mathrm{~mm}$ (2.0 in) diameter polyvinyl chloride (PVC) well casing, grouted into place using the same weak grout mix used for the inclinometer casing, housed the vertical SAA. To enable future retrieval of the SAA, silica sand was used to fill the annulus between the $25.4 \mathrm{~mm}$ (1.0 in) approximate diameter sensor array and the inner wall of the casing. The sand would later be jetted out with water to free the instrument. The fine sand backfill was placed by pouring from the top of the casing. The recommended installation method for the SAA now includes direct insertion into a $25 \mathrm{~mm}$ ( 1 in) inner diameter casing, which is grouted into place prior to the array installation [12]. This recommendation method had not been developed yet at the time of this installation. The consequential effect is the appearance of spurious displacements resulting from movement of the sand backfill rather than actual lateral movement of the clay deposit.

Beginning in April 2007, a $4.5 \mathrm{~m}$ (14.8ft) high, geosynthetic reinforced earth wall was constructed on the east bank of the Champlain Canal to mimic the load of the proposed bridge abutment, upon which an additional $1.5 \mathrm{~m}(4.9 \mathrm{ft})$ of fill was placed. With the surcharge in place, ground displacements began to accumulate and the lateral displacement of the foundation soils could be discerned. The zone of lateral squeeze can be seen in Figure 6 with displacements approaching $20 \mathrm{~mm}$ ( $0.79 \mathrm{in})$, from 3 to $5 \mathrm{~m}$ (9.8 to $16.4 \mathrm{ft}$ ) depth after April 2007. Figure 6 shows a comparison between the displacement measurements from a traditional inclinometer and the vertical SAA system for a three-month period of monitoring following the surcharge fill placement; that is, May 2007 is used as the zero reading. The trends from both methods of instrumentation are similar. The right side of Figure 6 shows the continuous displacement profile from the SAA system software for the four-month monitoring period after surcharge fill placement. Total displacements measured by both systems were less than $18 \mathrm{~mm}$ ( $0.71 \mathrm{in})$, but the general trends are discernible.

The horizontal SAA was installed after the PVDs had been driven, just prior to the construction of the surcharge embankment, approximately $5 \mathrm{~m}(17.5 \mathrm{ft})$ east of the westmost extent of the embankment and approximately $0.3 \mathrm{~m}$ $(1 \mathrm{ft})$ west of a row of PVDs. The array was pushed into ten sections of $25.4 \mathrm{~mm}$ ( 1 in) diameter PVC conduit, which had been glued together with PVC cement prior to the array insertion. Cable-pulling lubricant was used to assist the array insertion. However, the $32 \mathrm{~m}$ (104 ft) length was inserted into the full length of PVC conduit with relative ease even in spite of having to install the array against a slight upward grade. The array-conduit assembly was placed in a small trench, approximately $0.3 \mathrm{~m}(1 \mathrm{ft})$ deep, within a previously placed gravel drainage layer. The displaced drainage material was backfilled around the conduit. The initial position of the horizontal SAA was obtained by laptop connection within minutes of the installation. The earth station for wireless data collection was installed a few days later, coinciding with the start of the embankment construction. The horizontal SAA transmitted wireless data every four hours, after an initial evaluation period, where data was collected every hour.

Figure 7 shows the settlement profile from the horizontal SAA and a row of settlement plates (SP1, SP2, and SP3). This figure includes the horizontal SAA settlement data shown as a contour plot through February 2008, at which time the array was extracted prior to the pile installation at the site. The settlement plate profile is only provided through August 2007 in Figure 7 though it can be seen that the shape and values 


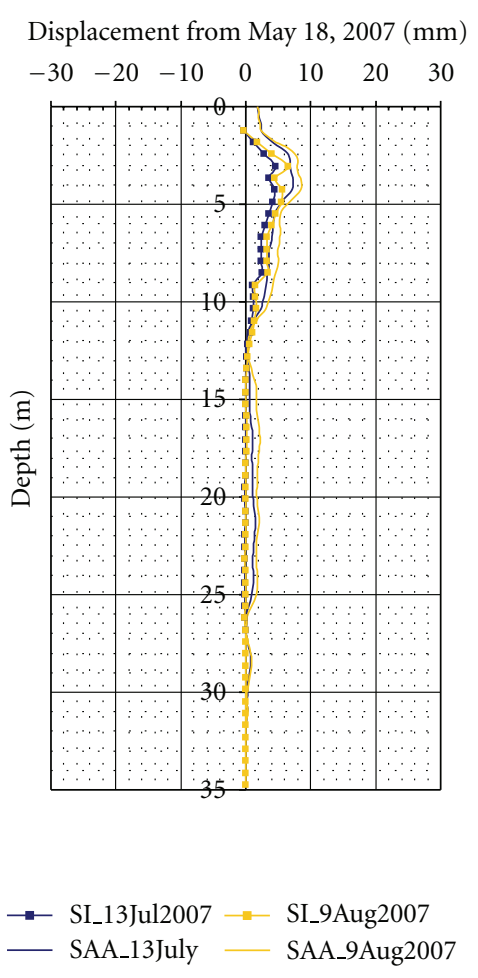

(a)

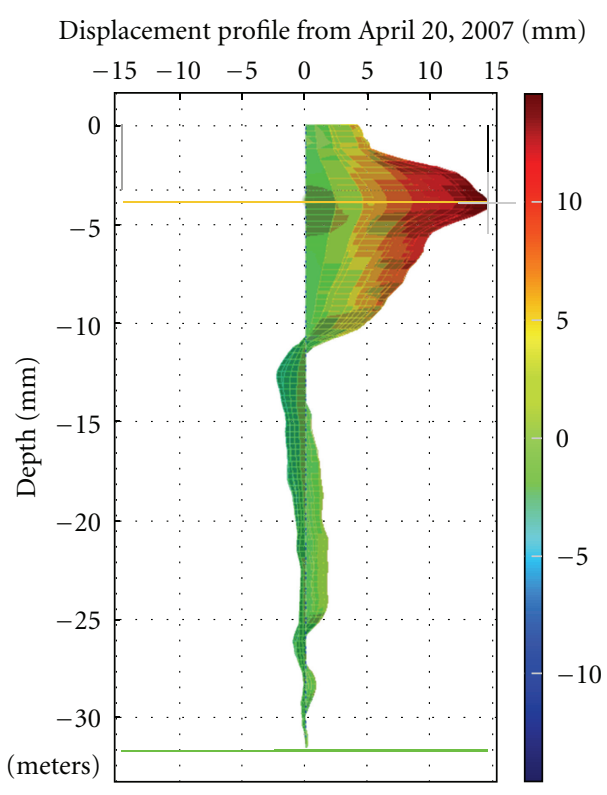

(b)

FIGURE 6: Comparison of vertical SAA and traditional slope indicator displacement data during surcharge loading.

of the profiles from both methods of instrumentation is quite similar. It can been seen from the time history plots of displacement in Figure 8 that the settlement plates (SP1, SP2, and SP3) experienced greater total settlement, approximately $280 \mathrm{~mm}$ (11.0 in) versus $225 \mathrm{~mm}$ ( 8.9 in) maximum observed SAA settlement. This difference is attributable to the fact that the settlement plates were located approximately $4 \mathrm{~m}(13.1 \mathrm{ft})$ east of the horizontal SAA, a location bearing more of the surcharge load. The $\mathrm{x}$-values shown for the SAA and the SPs correspond to the position of the measurement on Figure 7, measured from the cable end of the SAA.

Although the traditional site instrumentation was not ideally located for direct comparison with the vertical and horizontal SAA readings, this project demonstrates the usefulness of SAAs for construction monitoring. The information provided by these two SAA systems helped NYSDOT engineers evaluate the effectiveness of the geotechnical treatments utilized at this site, namely, surcharge loading and PVDs. Information from the horizontal installation, especially, helped engineers make decisions about the surcharge waiting period during construction. Specifically, the settlement profile beneath the embankment and the lateral squeeze of the underlying soft clay layer were available in real time. Had it been necessary, the construction schedule at this site might have been accelerated based on interpretation of the real-time settlement and rate of settlement information provided by the horizontal SAA. At the end of monitoring, both SAAs were successfully retrieved for reuse on other projects. The same methodologies applied at this site could be used for longer-term monitoring of foundation soils of permanent structures.

\section{SAA Field Installation at IJkdijk}

The IJkdijk (Dutch for "calibration levee") is a test site in The Netherlands for inspection and monitoring technologies for levees. The objectives of this site are two-fold: first to develop and validate new sensor techniques, and second to perform full-scale failure experiments on levees to understand their fundamental behavior. This should increase the quality of the levee inspection process and the safety assessment of levees. The final goal is to develop tools to respond to flood threats in a timely manner with appropriate measures.

The first task of this project was a full-scale consolidation test on an instrumented levee. Uncertainties regarding the bulk properties of a peat layer in the subsoil, based solely on laboratory testing, necessitated this field test to determine the permeability and the strength parameters in situ. In this full-scale test, one vertical SAA and one horizontal SAA were used as experimental instrumentation. Details of this test are given in [13]. In view of the accurate measurements obtained from the SAAs during this consolidation test, the SAA became the reference system for the evaluation of other deformation measurement systems in subsequent tests. The following presents the design and execution of the first large levee stability test.

The levee for the first production stability test at IJkdijk was constructed with a height of $6 \mathrm{~m}(19.7 \mathrm{ft})$, a length of 


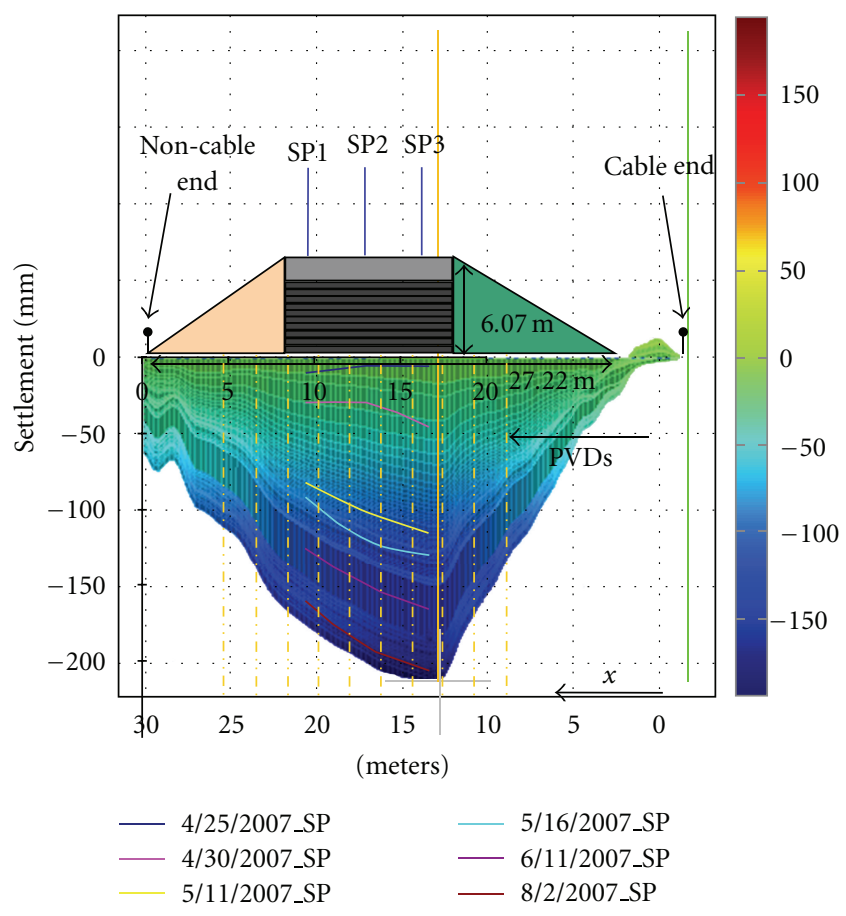

Figure 7: Settlement profile from horizontal SAA (contour plot) and nearest set of surface settlement plates (discrete lines).

$100 \mathrm{~m}(328.1 \mathrm{ft})$ and a base width of $27 \mathrm{~m}(88.6 \mathrm{ft})$, with a crest width of $3 \mathrm{~m}(9.8 \mathrm{ft})$ and side slopes of $1: 1.5(\mathrm{~V}: \mathrm{H})$ on the dry side and $1: 2.5$ on the wet side. The levee was built parallel to a local canal levee and on top of 1.3 to $3 \mathrm{~m}$ ( 4.3 to $9.8 \mathrm{ft}$ ) of clay and peat. The levee core is sand, with a thick clay cover. This is the usual configuration of new levees in The Netherlands. For this full-scale testing, using sand inside is an advantage since the levee can be filled with water, which reduces strength and increases the load on the subsoil. An aerial view of the levee on the second day of the test is shown in Figure 9.

To enable the calibration of the new techniques and to evaluate the test in general, reference monitoring systems including three vertical SAAs were installed in this stability test. Based on successful early field tests, the SAA system was deemed suitable as the reference system for monitoring the levee deformation. A cross-section of the levee showing all installed systems is shown in Figure 10. Some of the systems were installed along the length of the levee, but most of them were concentrated in three cross-sections, one in the middle and two $35 \mathrm{~m}(114.8 \mathrm{ft})$ away from the middle. To avoid damage from postconstruction installation, all tubes and buried cables were installed before and during the construction of the levee.

The loading sequence to bring the levee to failure is indicated in Figure 11 and consisted of six stages. First, the bathtub on the wet side was filled, followed by an excavation of $1 \mathrm{~m}(3.3 \mathrm{ft})$ on the other side. Second, the excavation was enlarged down to the sand base. In Figure 9, this phase had just started. Third, the sand core was filled to $2 / 3$ of its height with water. The fourth step was to drain the excavation. In

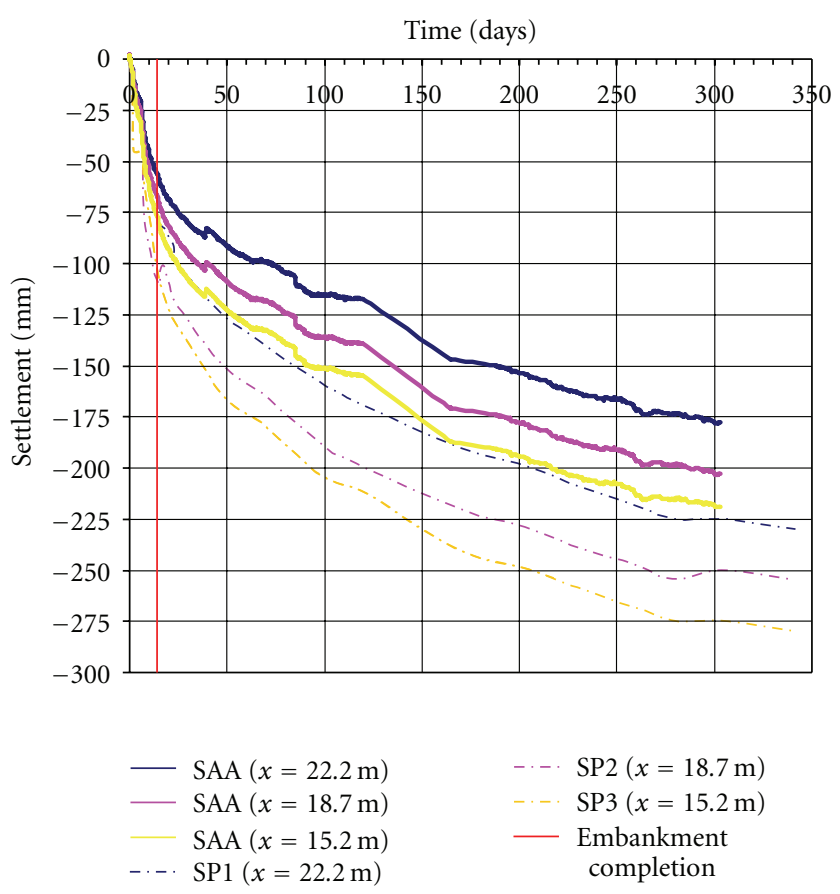

FIGURE 8: Time history of displacement from three SAA and surface settlement plate locations (consolidation data).

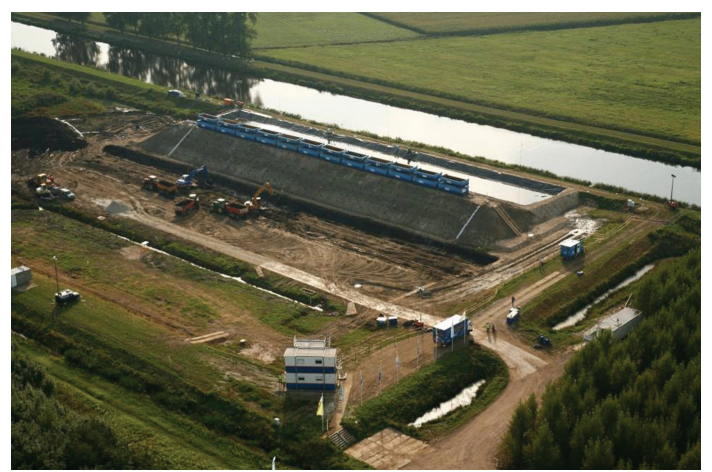

Figure 9: Aerial view of the stability test levee.

the fifth step, the containers on the crest were filled with water, and finally, in the sixth step, the sand core was filled completely, thus completing this sequence of internal and external loading.

The full-scale stability test began on September 25, 2008. As planned, the test started with the filling of the bathtub, closely followed by the shallow excavation. The second phase of the test, that is, deepening and widening of the excavation (Figure 9) was completed on the second day of the test. On the third day of the test, the filling of the sand core of the levee from within, through the built-in infiltration tubes, commenced. Because of the apparent variation in permeability, the pore pressures in the sand core increased rather irregularly. After nearly four hours, a section of about $30 \mathrm{~m}(98.4 \mathrm{ft})$ in length failed within approximately 40 seconds. One of the instrumented cross-sections was 


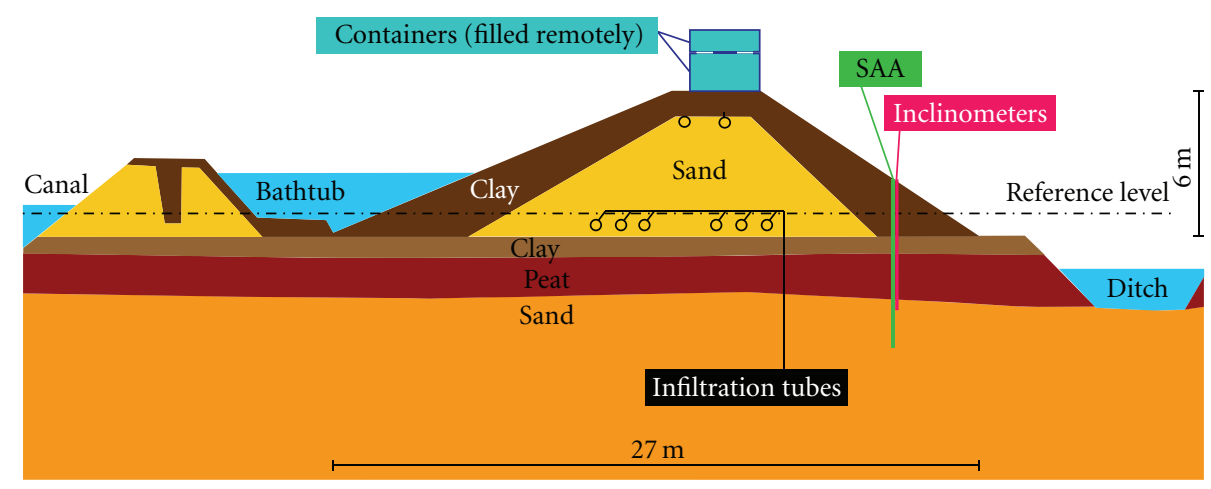

Figure 10: Cross-section of instrumented stability test levee.
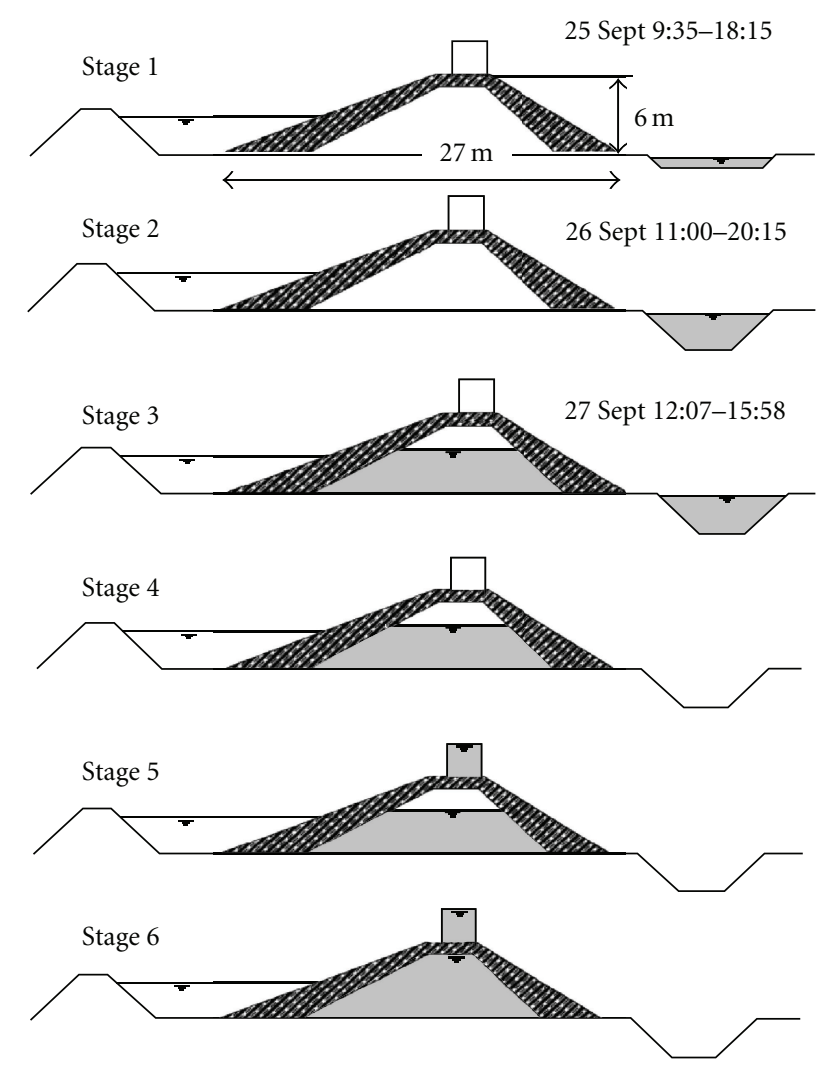

FIGURE 11: Six stages of loading sequence.

well within the part of the levee that failed. Figure 12 shows measurements from one of the vertical SAAs, with measurements at a $0.305 \mathrm{~m}(1 \mathrm{ft})$ intervals with depth.

The SAA was installed well below the slip plane. It can be seen that the levee was still moving because of consolidation as a result of the construction on the peat layer when the excavation was made. This caused an increase of deformations, which slowed down during the first night of the test. The enlargement of the excavation caused a large increase of deformations, which slowed down during the night. During the filling of the sand core, the deformations strongly increased until the clearly visible failure occurred, that is, the 27 Sep 16:04 data line in Figure 12. The failure

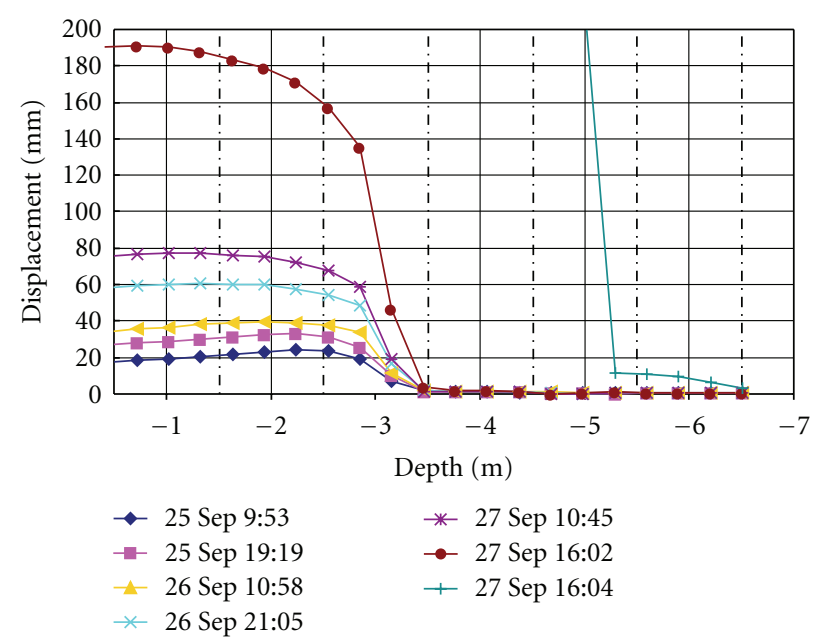

Figure 12: Lateral displacement measurements from SAA located in an instrumented cross-section at the middle of the test levee (center of levee shown in Figure 9).

caused such large movements, more than $3 \mathrm{~m}(9.8 \mathrm{ft})$, that the SAA was drawn out of its end anchor, resulting in failure of the subsoil that appeared to occur at $5.25 \mathrm{~m}(17.2 \mathrm{ft})$ depth (Figure 12) that is incompatible with other findings which showed failure occurring at around $3.5 \mathrm{~m}(11.5 \mathrm{ft})$ depth. However, the SAA continued to provide data through this large deformation and was retrieved for use in the next test. Figure 13 presents a photo of the levee immediately after it failed. The SAA measured deformations were confirmed by post test surveying measurements.

An additional excavation was made next to the middle instrumented cross-section, a few days after the failure. This showed that large cracks had appeared in the peat, which were filled by clay from the original surface layer. These cracks enabled the transport of infiltrated water down to a thin layer of about 5 to $15 \mathrm{~cm}$ (2.0 to 5.9 in) of sand between the peat layer and a thin, impermeable layer on top of the base sand, which appeared to be present only under a part of the levee, including the part which failed. Clear signs of sliding along this more or less horizontal sand layer were found as far as the forensic excavation was possible. 


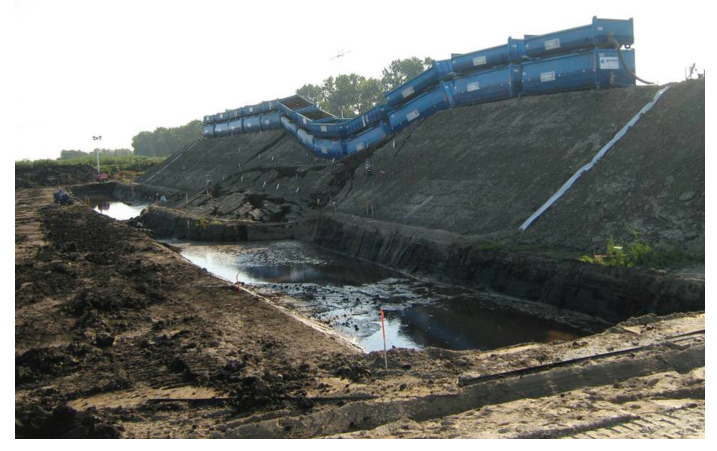

Figure 13: Levee after failure.

Although comparison plots are not available between the SAA and traditional displacement monitoring systems due to difficulties with the traditional system, this project demonstrates the usefulness of the SAA system for real-time monitoring of levees. The IJkdijk project has identified real-time information about the status of the water system and levees as an important precondition for large-scale water management systems.

\section{Local System Identification Analyses}

5.1. Field Instrumentation Strategy. Identification and calibration of a soil model solely using records of a surface motion or even the motion provided by one vertical array, (i.e., [15]) is a challenging task. This is especially the case if the system response is essentially multidimensional and marked by the development of large local deformations or interaction with structural elements. Thus, identification and model calibration activities using field data remain relatively scarce in view of a historical lack of appropriate data. The limited number of sensors commonly employed to monitor field sites often leads to open-ended indeterminate calibration and identification problems. Such problems require advanced three-dimensional instrument configurations, along with data reduction techniques, that go beyond usual and simple approaches. However, such instrumentation is limited due to prohibitive costs. The low cost of the SAA system provides a unique opportunity to monitor the response of complex soil and soil-structure systems using three-dimensional configurations.

In fact, the SAA is enabling a new strategy to monitor the static and dynamic response of soil and soil-structure systems. This array allows easy three-dimensional instrumentation of new and existing geotechnical systems with a dense network of accelerometer and deformation sensors. In view of their small size, these sensors may be installed at virtually any location within a system and along its boundaries without compromising the system's structural integrity. Figure 14 presents a sketch rendering this vision using a number of SAAs installed to monitor level ground, slope, and soil-pile interaction at a bridge abutment site where the soil slides and deforms due to extreme loading (traffic loads, earthquakes, rain fall, etc.). Such comprehensive dense instrumentation enables a new and more efficient local identification methodology, as described below.

5.2. Local System Identification (SI) Algorithm. The local identification technique capitalizes on the dense measurements provided by the SAA. This algorithm (Figure 15) consists of the following steps: (1) evaluation of strain tensor time histories using the static and dynamic motions recorded by a cluster of closely spaced sensors, (2) estimation of the corresponding stress tensors utilizing a preselected class of constitutive models of soil response, (3) computation of the deformations or accelerations associated with the estimated stress tensors using the equilibrium equations, and (4) calibration and evaluation of an optimal model of soil response. This approach focuses on the analysis of local soil dynamic characteristics and properties without interfering with the boundary conditions or adjacent response mechanisms [15, 16].

\section{Proof-of-Concept Using Two-Dimensional (2D) Soil Systems}

The capabilities of the SI algorithm were assessed using a number of computer simulations along with analyses of centrifuge test data of small-scale soil systems with sensor configurations that mimics those enabled by the SAA. The performed simulations addressed the identification of the complex response of a soil system behind a retaining wall, as shown in Figure 16. These simulations showed that the local SI technique provides an effective means to analyze the constitutive behavior of complex, massive soil and soilstructure systems at specific locations independently of adjacent response mechanisms or material properties. For the $2 \mathrm{D}$ problem of Figure 16, the motion recorded by a $3 \times 3$ (or larger) cluster of accelerometers and inclinometers may be used to identify low and large strain dynamic properties of the soil comprised within the instrumented zone independently of adjacent soil (even for a complex multilayered site). For instance, a subset of $5 \times 5$ accelerometers of the soil system shown in Figure 16 were efficiently used to identify the lowstrain shear modulus, $G_{0}$, of an intricate zone of this system [17].

The centrifuge tests were conducted under a 50 g gravity field for the clay soil retaining structure system shown in Figure 17 [16]. A one-dimensional lateral shaking was imparted along the model base. The $2 \mathrm{D}$ response of the clay soil was monitored at 15 locations behind the retaining structure using a $5 \times 3$ array of traditional accelerometers. The recorded accelerations provided ample experimental data to locally assess the constitutive stress-strain relationship of the clay layer using the SI algorithm. A multisurface plasticity technique was used to idealize the nonlinear and path-dependent stress-strain behavior of the clayey soil. The identified accelerations at location (4 and 2) are shown in Figure 18, along with the corresponding shear modulus variation with strain amplitudes. Good agreements were obtained between computed and recorded accelerations at 


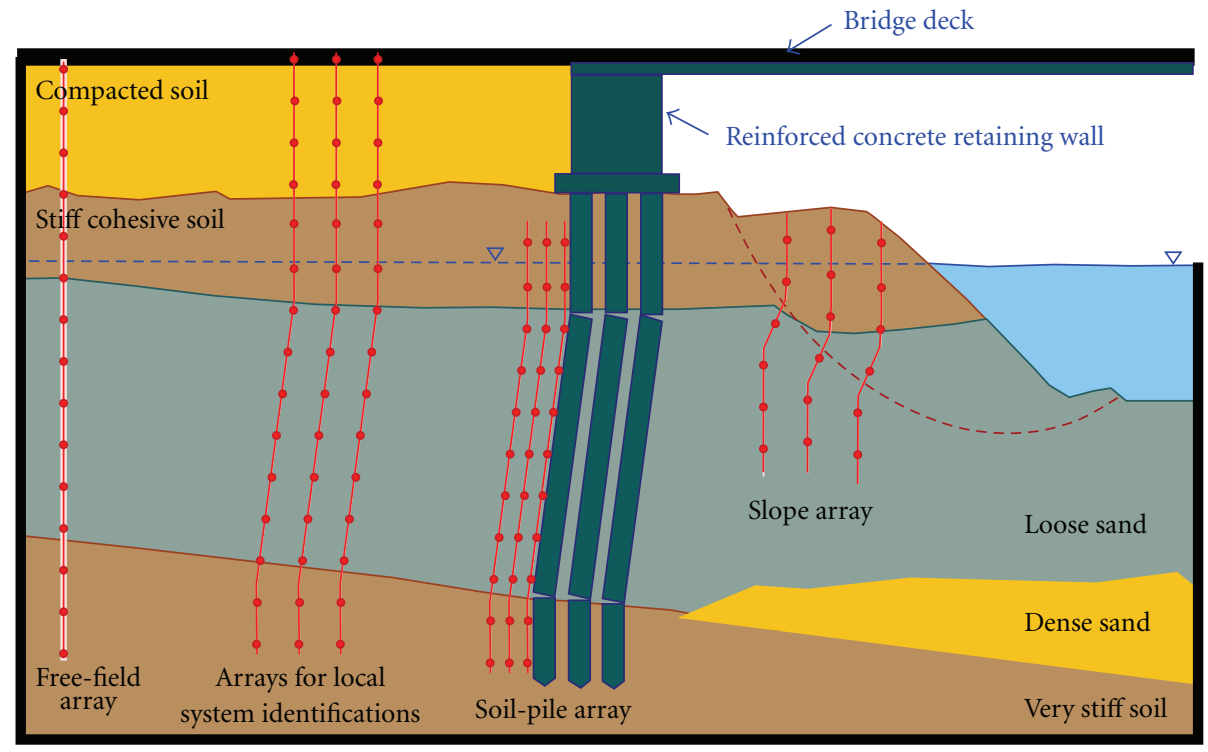

FIGURE 14: Sketch rendering the vision of dense local instrumentation of active soil and soil-structure systems using shape acceleration arrays (SAAs).

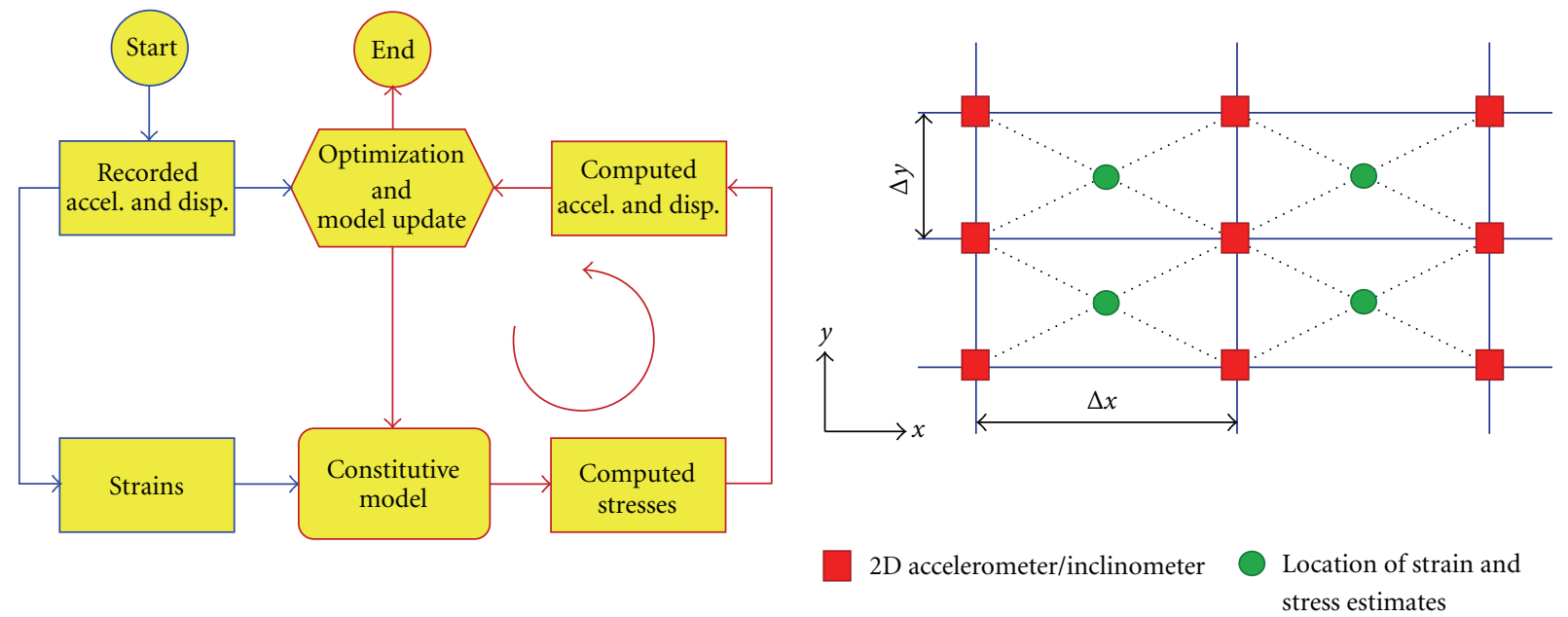

FIGURE 15: Algorithm of the developed local system identification technique and detail of instrument configuration for a $2 \mathrm{D}$ local identification analysis.

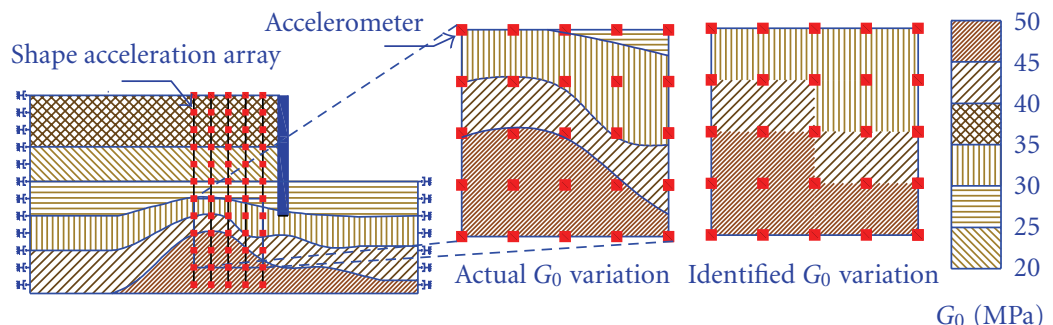

FIGURE 16: Sample results of a numerical simulation conducted to show the potential of using shape acceleration arrays (SAAs) to identify locally the low-strain mechanical properties of multilayered sites or other complex soil systems [14]. 


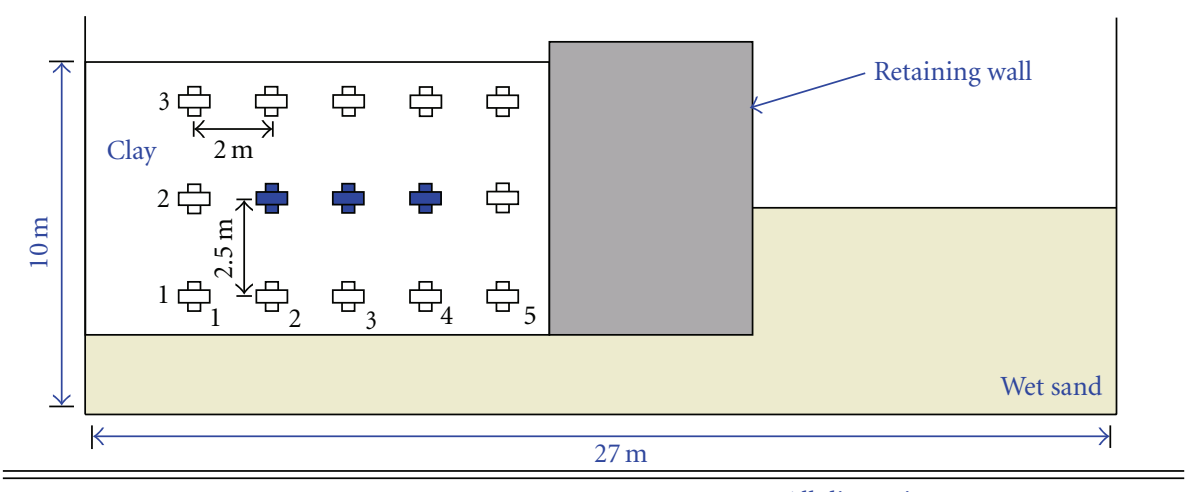

All dimensions are prototype

号臬2D acceleromteres

FIGURE 17: Configuration of analyzed soil-retaining wall centrifuge model.

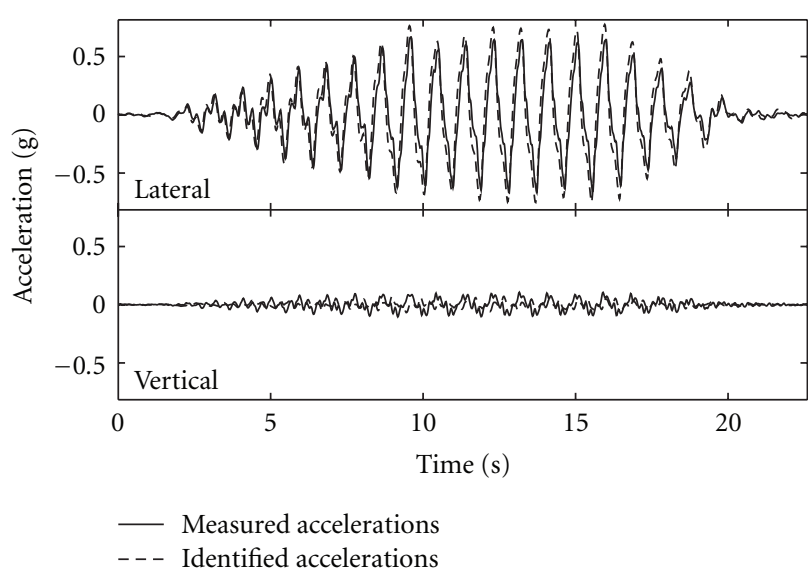

(a)

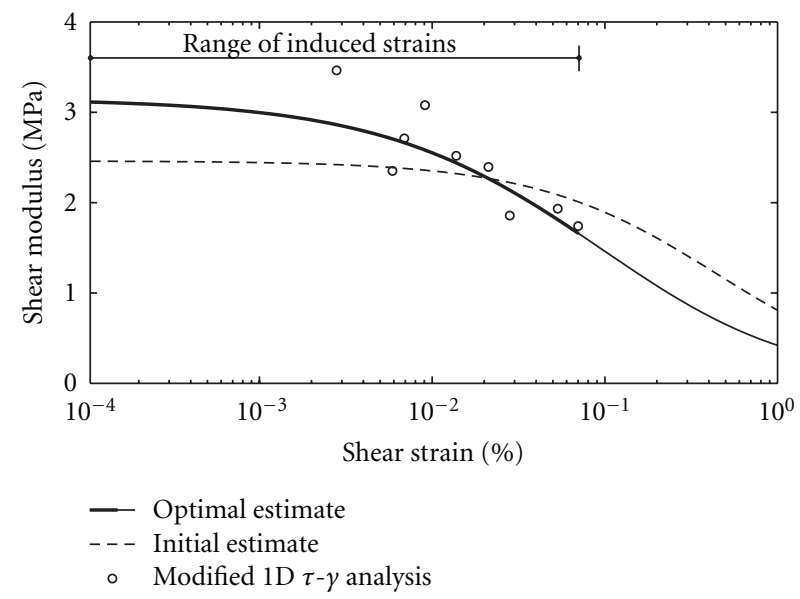

(b)

FIGURE 18: Local identification of the retaining wall-soil centrifuge model of Figure 17 when subjected to a strong shaking (acceleration time histories and shear moduli at the $(4,2)$ instrument location).

this and other locations. The modified one-dimensional stress-strain analysis takes into account the impact of lateral normal stresses rather than only using a shear beam idealization $[14,16]$.

6.1. Three-Dimensional Site Characterization. The newly developed SAA and local identification approach are currently being used to develop an effective new approach for site characterization. A network of SAAs has been installed (spring 2009) at the Wildlife Refuge free field site in California. This network has a three-dimensional configuration. Such a configuration will enable the development of improved tools to: (1) characterize the 3D response of field sites and other geotechnical systems, (2) accurately evaluate the in situ small-strain and nonlinear mechanical properties of these systems, and (3) calibrate soil models. More specifically, the set of installed arrays will be used to fully characterize and identify the soil mass comprised within the sensors. The data provided by the SAAs and associated data reduction tools will produce significantly more and better information than current soil sample experiments, with the added benefit that this information is for in situ conditions and covers continuous soil strata from the ground surface up to $100 \mathrm{~m}(328 \mathrm{ft})$ depth and that the issues of soil sample disturbance and size are circumvented.

\section{Conclusions}

This paper presented two successful field applications of the shape acceleration array (SAA) system, at an active bridge realignment site on a $30 \mathrm{~m}$ deposit of very soft clay and a full-scale levee testing facility in The Netherlands, which demonstrates how this system could be utilized for real-time health monitoring of civil infrastructure. A new local identification technique to characterize the response and assess the properties of soil and soil-structure systems was also presented. The developed identification technique provides an effective tool to locally analyze and assess the static and 
dynamic response of soil and soil-structure systems using the acceleration and deformation measurement provided by the SAA. This technique does not require the availability of boundary condition measurements, or solution of a boundary value problem associated with an observed system. Studies are planned to capitalize on the capabilities of the SAA and identification technique to analyze the mechanisms of large deformation and lateral spreading of soil and soilstructure systems.

\section{Acknowledgments}

The array development was partially funded by the National Science Foundation (NSF) sensor program (Grant no. CMS0330043) and is manufactured by Measurand, Inc. This NSF support is gratefully appreciated. The authors wish to thank Lee Danisch and Terry Patterson of Measurand, Inc. for their contribution to these projects, including their efforts toward all the field installations. The authors would also like to express their gratitude to the engineers, drillers, and maintenance staff who participated in these field installations, without whom this research would not have been possible. The authors would like to acknowledge the contribution of Dr. C. Oskay and Dr. A. Elmekati to the development of the local system identification technique.

\section{References}

[1] American Society of Civil Engineers, "Raising the grades: small steps for big improvements in america's failing infrastructur," Report Card for America's Infrastructure, January 2008, http://apps.asce.org/reportcard/2005/actionplan07.cfm.

[2] K. Arulanandan and R. F. Scott, Eds., Verification of Numerical Procedures for the Analysis of Soil Liquefaction Problems, vol. 1, Balkema, Davis, Calif, USA, 1993.

[3] National Research Council, Liquefaction of Soils During Earthquakes, Committee on Earthquake Engineering, Washington, DC, USA, 1985.

[4] K. Ishihara, Soil Behaviour in Earthquake Geotechnics, Oxford University Press, New York, NY, USA, 1996.

[5] R. F. Scott, "Lessons learned from VELACS project," in Proceedings of International Conference on the Verification of Numerical Procedures for the Analysis of Soil Liquefaction Problems, K. Arulanandan and R. F. Scott, Eds., pp. 773-1784, A.A. Balkema, Davis, Calif, USA, October 1994.

[6] A. J. Whittle, "Why we need improved soil models," in Proceedings of A Symposium in Honor of Robert V. Whitman, pp. 59-75, Massachusetts Institute of Technology, 1995.

[7] R. B. Peck, "Liquefaction potential: science versus practice," Journal of Geotechnical Engineering ASCE, vol. 105, no. 3, pp. 393-398, 1979.

[8] T. Abdoun, V. Bennett, L. Danisch, T. Shantz, and D. Jang, "Field installation details of a wireless shape-acceleration array system for geotechnical applications," in Sensors and Smart Structures Technologies for Civil, Mechanical, and Aerospace Systems, Proceedings of SPIE, San Diego, Calif, USA, March 2007.

[9] V. Bennett, T. Abdoun, T. Shantz, D. Jang, and S. Thevanayagam, "Design and characterization of a compact array of MEMS accelerometers for geotechnical instrumentation,"
Smart Structures and Systems Journal, vol. 5, no. 6, pp. 663679, 2009.

[10] T. Abdoun, V. Bennett, R. Dobry, S. Thevanayagam, and L. Danisch, "Full-scale laboratory tests using a shapeacceleration array system," in Proceedings of the 4th Decennial Geotechnical Earthquake Engineering and Soil Dynamics Conference, Geotechnical Special Publication, Sacramento, Calif, USA, May 2008.

[11] R. Dobry, S. Thevanayagam, C. Medina et al., "Mechanics of lateral spreading observed in a full-scale shake test," Journal of Geotechnical and Geoenvironmental Engineering, vol. 137, no. 2, pp. 115-129, 2011.

[12] T. Abdoun, V. Bennett, L. Danisch, and M. Barendse, "Realtime construction monitoring with a wireless shape-acceleration array system," in Proceedings of the GeoCongress Characterization, Monitoring, and Modeling of GeoSystems, Geotechnical Special Publication, pp. 533-540, New Orleans, La, USA, March 2008.

[13] M. A. Van, C. Zwanenburg, A. R. Koelewijn, and H. van Lottum, "Evaluation of full scale levee stability tests at booneschans and corresponding centrifuge tests," in Proceedings of the 17th International Conference on Soil Mechanics and Geotechnical Engineering, M. Hamza, M. Shahien, and Y. ElMossallamy, Eds., Alexandria, Egypt, October 2009.

[14] M. Zeghal and C. Oskay, "A local identification technique for geotechnical and geophysical systems," International Journal for Numerical and Analytical Methods in Geomechanics, vol. 27, no. 11, pp. 967-987, 2003.

[15] M. Zeghal, A. W. Elgamal, H. T. Tang, and J. C. Stepp, "Lotung downhole array. II: evaluation of soil nonlinear properties," Journal of Geotechnical Engineering, vol. 121, no. 4, pp. 363378, 1995.

[16] C. Oskay, Local identification analyses of soils and soil-structure systems, Ph.D. thesis, Rensselaer Polytechnic Institute, Troy, NY, USA, 2003.

[17] A. Elmekati, A Local-Global scheme for Identifying the Nonlinear Response of Geotechnical Systems, Ph.D. thesis, Rensselaer Polytechnic Institute, Troy, NY, USA, 2007. 

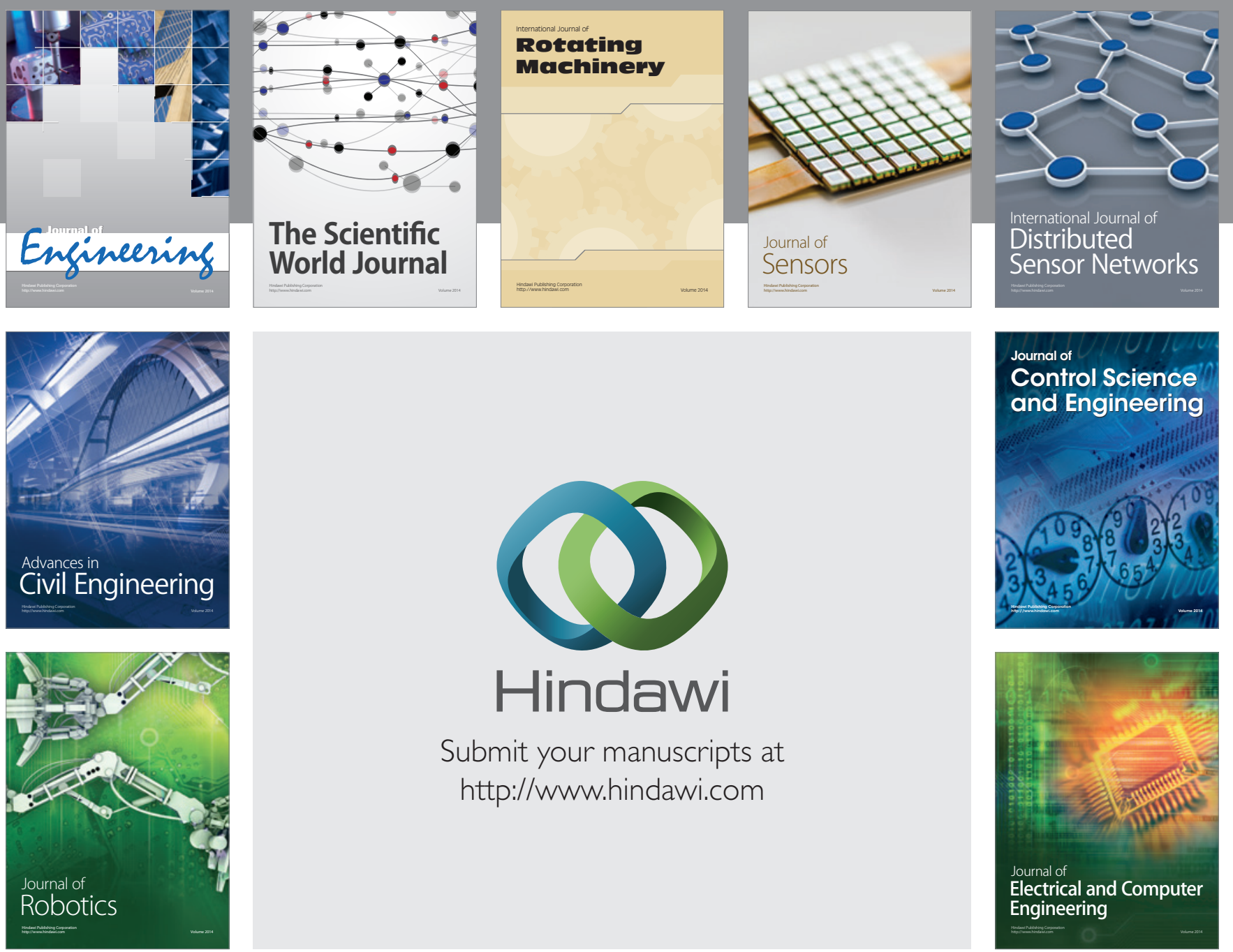

Submit your manuscripts at

http://www.hindawi.com
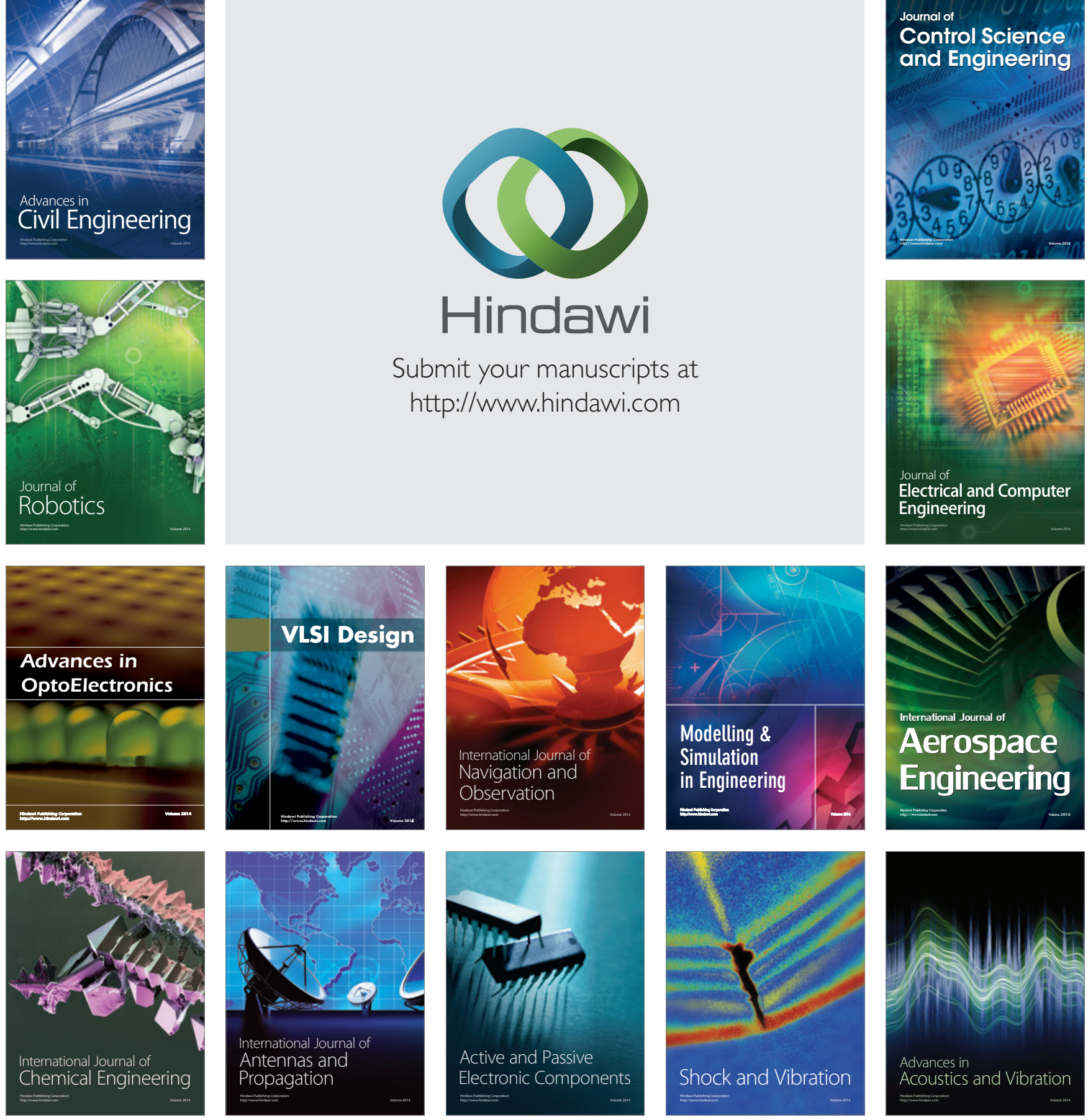